\title{
Possible Effect of Human-Experimenter on Homeopathic-Like Aqueous Preparations
}

\author{
Vladimir Korenbaum ${ }^{1,+}$, Tatiana Chernysheva ${ }^{2}$, Victorya Galay ${ }^{2}$, Roman Galay ${ }^{2}$, Alexandr Ustinov ${ }^{3}$, \\ Sergei Zakharkov ${ }^{1}$ and Nikolai Bunkin ${ }^{4, *}$ (D) \\ 1 V.I. Il'ichev Pacific Oceanological Institute, Far Eastern Branch of Russian Academy of Sciences, \\ 43 Baltiiskaya Str., 690041 Vladivostok, Russia; v-kor@poi.dvo.ru (V.K.); k-v-i_2006@mail.ru (S.Z.) \\ 2 Clinics of Functional Medicine "Manus", 1-st Morskaya Str., 4, 690007 Vladivostok, Russia; \\ tanyasavod@gmail.com (T.C.); manus.prim@gmail.com (V.G.); galay.fam@gmail.com (R.G.) \\ 3 Institution of Chemistry, Far Eastern Branch of Russian Academy of Sciences, 100-Years of Vladivostok \\ Avenue, 159, 690022 Vladivostok, Russia; chemi@ich.dvo.ru \\ 4 Bauman Moscow State Technical University, 2-nd Baumanskaya Str., 5/1, 105005 Moscow, Russia \\ * Correspondence: nbunkin@mail.ru \\ + The author has passed away.
}

Citation: Korenbaum, V.;

Chernysheva, T.; Galay, V.; Galay, R.;

Ustinov, A.; Zakharkov, S.; Bunkin, N.

Possible Effect of

Human-Experimenter on

Homeopathic-Like Aqueous

Preparations. Water 2021, 13, 1475.

https://doi.org/10.3390/w13111475

Academic Editor: Elmar C. Fuchs

Received: 9 April 2021

Accepted: 19 May 2021

Published: 24 May 2021

Publisher's Note: MDPI stays neutral with regard to jurisdictional claims in published maps and institutional affiliations.

Copyright: (C) 2021 by the authors. Licensee MDPI, Basel, Switzerland. This article is an open access article distributed under the terms and conditions of the Creative Commons Attribution (CC BY) license (https:// creativecommons.org/licenses/by/ $4.0 /)$.

\begin{abstract}
Homeopathy is one of the applications in structured water influencing human health. The objective is to search for the physical basis of homeopathy. This methodology includes a study of absorption in the far IR spectral range, absorption and refraction in THz diapason, dynamic light scattering in the UV-near IR spectral range for the blinded samples of homeopathic-like preparations (HLP) of several parent substances and hidden/apparent controls, and statistical analysis on the significance of distinctions in spectral data between ensembles of HLP of each parent substance and ensembles of hidden/apparent controls. The analysis of nine independent blind studies of aqueous HLP of several parent substances gave statistically significant spectral differences in some preparations with an apparent control (25 comparisons of 35$)$ and a hidden control (11 comparisons of 40). The revealed dominance in the occurrence frequency of differences in any HLP with an apparent control can be treated as involving most of these changes to the samples by the spectral measuring process. This allows interpreting the main mechanism of manifestation of the spectral changes found as the "observer effect". The therapeutic effect of HLP may be assumed as a combination of the "observer effect" from the physician side and a "placebo effect" from the patient side.
\end{abstract}

Keywords: water; spectral measurements; homeopathic-like preparations; blinded study; statistical analysis; physical interpretation; scientific basis

\section{Introduction}

Traditional homeopathy propounded in 1796 by Samuel Hahnemann is widely used for curation. It may be interpreted as one of the applications of influencing structured water on human health. However, in recent years, more and more criticism has been expressed regarding homeopathy from current (allopathic) medicine and biology. The criticism is based on insufficient evidence of the effect of therapy with homeopathic preparations, as well as on the lack of scientific basis. Some authors (see, for example, [1]) argued that the clinical effect of homeopathy is nothing more than a placebo effect, i.e., a result of auto-suggestion of the patient. The statements of this type are actively disputed by complementary/alternative medicine (CAM) authorities (see, for example [2]). At the same time, homeopathic medicines continue to be actively used by patients in many countries, which is difficult to explain by only the marketing efforts of the manufacturers. However, let us leave the area of evidencing therapeutic efficacy of homeopathic remedies and try to focus on the problem of physical justification of homeopathy, which is no less intensively discussed [3-6]. 
The traditional way to prepare homeopathic remedies (dilution/potentiation of parent substances in substrate) prevents the design of double-blind experiments for rigorous study of properties of these medicines, because the condition of the identity of the substrate is violated for samples corresponding to various parent substances.

Meanwhile, other types of structured water applications-homeopathic-like preparations, which are frequently declared as "electronic copies" or so-called "nosodes" obtained by "imprinting" of parent substance onto aqueous or other substrate-have also received certain popularity in CAM. A number of firms (Meripharm GmbH DE, Metabolics UK, Epigenetics UK, Life-Work Potential Ltd USA, Imedis RU) are producing broad kits of "diagnostic nosodes". "Electronic copying", at least with aqueous solutions, as a non-contact procedure, allows to achieve an identity of a substrate for all analyzed samples, and thereby to design a blinded and randomized procedure to study homeopathic-like preparations, which meets high-level research standards.

Several types of devices for "electronic copying" are available now. The first available was the "radionic" magneto-geometric copying apparatus, invented by M. Rae [7]. Simulator (Metabolics Ltd, Devizes, UK) is a version of M. Rae's apparatus, which is widely used in CAM. Other "electronic copying" techniques were invented later (see, for example, $[8,9]$ ).

Owing to the vagueness surrounding the physical basis of the "electronic copying" mechanism, the "electronic copying" instrument should be treated only as a "black box" (in the cybernetic sense) that could change the physical properties of water preparations.

One approach to study physical changes in preparations made with the "black box" is observing wide spectral bands of the samples [10]. However, the procedure must be strictly double-blinded in order to avoid possible human bias [1,11].

The aim of this study is a three-diapason spectral analysis of the samples of homeopathiclike preparations, accompanied by statistical analysis of the spectral data and physical interpretations of results for searching for the physical basis of homeopathy.

\section{Materials and Methods}

The research methodology included:

- 3-diapason (absorption in far infrared, absorption and refraction in terahertz, dynamic light scattering in ultra-violet-near infrared) spectral analysis of samples of homeopathic-like preparationsof several parent substances and hidden/apparent controls;

- Statistical analysis of distinctions in amplitudes of spectral data between ensembles (9-15 samples) of homeopathic-like preparations of each parent substance and ensembles (9-15 samples) of hidden/apparent controls;

- Evaluation of an occurrence frequency of the significant distinctions in 3 spectral diapasons with the estimation of a significant difference between hidden and apparent controls.

\subsection{Design of Experiments}

To provide the blind spectral study, the following procedure was developed:

(1) Voluntary numbering of sealed fabric vials with water substrate or empty containers filled with water substrate taken from one well-mixed portion, making the samples with the "black box", packing samples, writing and sealing the protocol of preparations (number of the sample-corresponding parent substance);

(2) Handling packed samples;

(3) Recording spectral files of each sample;

(4) Sorting spectral files of samples by preparations (parent substances) according to the firstly unsealed protocol of preparations;

(5) Statistical analysis of spectral data by preparations (parent substances).

Only step (1) of this procedure was not blinded, thus being dependent on the voluntary impact of the human experimenter, preparing homeopathic-like samples, while steps (2 and 3) were double-blind. Thus, an impact of the human-experimenter was excluded here. 
Steps (4 and 5), although fulfilled with the unsealed protocol of preparations, were strictly predetermined. Consequently, the impact of the human experimenter was minimized. According to this procedure, in all experiments, homeopathic-like samples and samples of hidden control "hc", representing a "blank" water substrate, were prepared and analyzed. All samples were randomly numerated, and the human experimenter did not know which preparation spectrum was currently being measured and recorded.

An additional not-blind procedure was applied to prepare samples of apparent control in some experiments. The container with each sample was labeled as " $K$ " at step (1). During step (1), these samples were prepared in analogy with "hc" samples (excluding numbering). However, unlike "hc" preparations (numbered randomly), the human experimenter unambiguously knew that sample " $K$ " was a "blank" water substrate, when he measured its spectrum in step (3). The samples " $\mathrm{K}$ " were voluntarily (by the human experimenter measuring spectra) distributed through the procedure of the experiment.

\subsection{Preparation of Samples}

The procedure of producing homeopathic-like preparations was described in detail in [12]. The numbering of vials/containers, corresponding to various parent substances, was written in the list (protocol), which was not revealed before completing spectral measurements (saving text files). The preparation procedure was carried out with the Simulator (Metabolics Ltd, Devizes, UK) apparatus by operators V.G. and R.G., who did not participate in further spectral analysis and processing.

Parent substances to prepare studied homeopathic-like samples were chosen empirically. They were:

- Hydroplasma - preparation for tissue repair (Vitaest, Almaty, Kazakhstan);

- Lydocaine-standard pharmacological solution;

- Alloplant-stimulator of vasculogenesis (Russian Center for Eye and Plastic Surgery, Ufa, Bashkortostan, Russia);

- Epstein-Barr virus-homeopathic remedy (Meripharm GmbH, Baden-Baden, Germany);

- Chorionic gonadotropin-standard pharmacological drug;

- Euphyllin—standard pharmacological solution Aminophylline;

- Analgin—standard pharmacological solution Metamizole sodium;

- Uranium-homeopathic remedy (Metabolics Ltd, Devizes, UK);

- Cerebral spinal fluid-homeopathic remedy (Metabolics Ltd, Devizes, UK).

The medical saline water solution or medical grade distilled water were used as a water substrate in various experiments.

Each sample was wrapped in aluminum foil to prevent against external electromagnetic fields, and all samples for every experiment were placed in a common package. The package was transported by a courier to the place of spectral analysis. The courier tried to keep the package away from household sources of electromagnetic fields (computers, televisions, mobile phones). About 5-10 days elapsed between the homeopathic-like preparations-making procedure and the completion of spectral analysis for terahertz spectra and dynamic light scattering spectra, and there was a delay of 20-25 days for the infrared spectra due to a longer procedure of preparing cuvettes and, therefore, lower measuring speed.

After completing the spectral analysis in each experiment, the spectral readings (text files) of individual samples were recorded and sent by email to the human experimenter V.K. (did not participate in producing preparations and spectral measurements of samples). He sorted spectra (spectral readings or spectral parameters) by parent substances in accordance with the first-time unsealed numbering protocol of preparations. The same human experimenter fulfilled the statistical processing of results by means of a predetermined algorithm [12].

In total, 9 independent experiments were made with a slightly varied list of preparations. 


\subsection{Spectral Measurements}

\subsubsection{Infrared Absorption}

Far infrared transmission spectra were measured with infrared Fourier spectrometer IRAffinity-1 (Shimadzu, Kyoto, Japan). Analysis was applied in the $400-4000 \mathrm{~cm}^{-1}$ (3000-25,000 nm) band, a resolution of $4 \mathrm{~cm}^{-1}$, with the number of scans at 30 . The transmittances $(\mathrm{T} \%)$ were calculated.

Measurements were made with an assembled cuvette with windows made of calcium fluoride $\left(\mathrm{CaF}_{2}\right)$, separated by a lead gasket with a thickness of $0.025 \mathrm{~mm}$, the internal cavity of which contained the test solution, placed with a disposable syringe. Before each measurement, the window and the gasket were washed with medical-grade distilled water and dried with filter paper.

In each of the 3 experiments, 15 samples of homeopathic-like preparations of parent substances-hydroplasma, lydocaine, alloplant, Epstein-Barr virus, chorionic gonadotropiand 15 samples of hidden control "hc" were studied. Twelve samples of apparent control " $\mathrm{K}$ " were additionally used only in one experiment. All samples were sealed in 5-ml glass ampoules containing medical-grade distilled water or saline solution ( 9 boxes by 10 items), purchased from one manufacturer. Samples were analyzed in random order. Each ampoule was opened just before the spectral measurement.

\subsubsection{Terahertz Absorption and Refraction}

Terahertz spectra were measured with spectrometer TPS Spectra 3000 (Teraview, Cambridge, UK). The method THz-TDS was used; this technique provides simultaneous evaluating spectra of transmission and refraction indexes. Measurements were made in the spectral band of 1.2-120 $\mathrm{cm}^{-1}$ with the resolution of $4 \mathrm{~cm}^{-1}$. The Fourier transform of the measured time functions of the electric field intensity into the spectra was performed using the apodization function Blackmann-Harris 3. Averaging over 1800 scans was made. The procedure is detailed in [13]. The temperature of all solutions during measurement was stabilized at $25 \pm 0.5^{\circ} \mathrm{C}$ by placing the cuvette in a thermostatic holder with a high-stability temperature controller 4000 Series (Specac Inc., Fort Washington, PA, US).

In each of three independent experiments, transmission spectra of electronic copies of lydocaine, euphyllin, analgin, and hidden control "hc" were studied. The apparent control " $\mathrm{K}$ " samples were prepared and used in two experiments. All samples were sealed in $2 \mathrm{~mL}$ plastic Safe-Lock containers (Eppendorf, Hamburg, Germany), filled with medical saline solution from one well-mixed portion. Samples were analyzed in a random order. Each container was opened just before the spectral measurement.

The substance from every container was collected in a disposable $5 \mathrm{~mL}$ syringe, from which both cuvettes were filled. The cuvettes were washed as follows. The measured sample was pumped out of the cuvette with the same syringe until it was visually dried. The next sample was pumped through the cuvettes in a volume of approximately $0.5 \mathrm{~mL}$ with a new syringe, then again, the entire sample was pumped out with this syringe. Then, the cuvette was finally filled with the sample intended for the next measurement.

\subsubsection{Dynamic Light Scattering}

The measurement consisted of the illumination of test samples with a laser (wavelength $633 \mathrm{~nm}$, intensity $2 \mathrm{~mW}$ ) and subsequent analysis of the autocorrelation function of the scattered radiation at an angle of $173^{\circ}$. The measurements were performed with Zetasizer nano ZSt (Malvern, UK) at temperature $25 \pm 0.1^{\circ} \mathrm{C}$.

The spectral characteristics of dynamic light scattering were determined directly in individual disposable cuvettes CUVETTE SQ 4SIDE 4ML (Sarstedt, Germany). The samples were poured into individual disposable cuvettes using micropipettes with disposable plastic nozzles.

The size of scatterers was calculated under the assumption of their sphericity. The spectral parameters "\% Intensity", "Size", and "St Dev (Size)" were evaluated. Based on 
the results of the spectra construction, spectra with the "Size" of the scatterers in a certain range were selected, while other samples were excluded.

There were 3 independent experiments in which refraction spectra were studied for homeopathic-like preparations of 3 parent substances from the list of lidocaine, euphyllin, analgin, uranium, cerebral spinal fluid, and hidden control "hc". The apparent control " $\mathrm{K}$ " samples were prepared and used in two experiments. Samples were analyzed in random order. Each disposable cuvette was opened just before the spectral measurement.

\subsection{Statistical Analysis}

During statistical processing, each spectral reading (or spectral parameter) for the ensemble of every homeopathic-like preparation was compared with the same spectral reading or parameter of the ensemble of hidden control orthe ensemble of apparent control by means of the Mann-Whitney non-parametric U-test (STATISTICA Advanced, StatSoft Inc.). The specially developed macros provided processing of files with the number of lines up to 2000. Spectral readings (wavelengths) or parameters were automatically fixed where statistical significance of differences (2-sided exact $p<0.05$ ) appeared. The direction (sign) of differences between ensembles was assessed by the sign of Z-distribution approximating Mann-Whitney statistics.

To meet Bonferroni's multiple comparison problem, for example when comparing $\mathrm{N}$ pairs of spectral readings between ensembles, a level of statistical significance should be $\mathrm{p}<\mathrm{p}_{\mathrm{B}}=0.05 / \mathrm{N}$. Therefore, statistically significant differences in the spectra were accepted if the condition in Equation (1) was satisfied for some adjacent wavelengths, where a Mann-Whitney $\mathrm{p}<0.05$ of the same sign was found [12].

$$
\mathrm{p}_{\text {cum }}=\mathrm{p}_{1}{ }^{*} \mathrm{p}_{2}{ }^{*} \ldots{ }^{*} \mathrm{p}_{\mathrm{n}}<\mathrm{p}_{\mathrm{B}}
$$

where $\mathrm{p}_{\text {cum }}$-cumulative statistical significance; $1,2, \ldots, \mathrm{n}$-numbers of adjacent wavelengths $\left(\mathrm{cm}^{-1}\right)$, for which the statistically significant difference Mann-Whitney $\mathrm{p}<0.05$ in the spectral reading between ensembles was revealed. These differences at adjacent wavelengths $1,2, \ldots, \mathrm{n}$ should have the same direction (sign) to be included in the Equation (1).

The central wavelengths $\lambda_{\text {centr }}$ of spectral bands $\left(\lambda_{1}-\lambda_{n}\right)$ characterized by statistically significant differences, in addition to the scale of $\mathrm{cm}^{-1}$, were also presented in the scale of $\mathrm{nm}$, according to the ratio $\lambda(\mathrm{nm})=10^{7} / \lambda\left(\mathrm{cm}^{-1}\right)$. In accordance with the ratio $1(\mathrm{eV})=8066\left(\mathrm{~cm}^{-1}\right)=1240(\mathrm{~nm})$, the wavelength describes the spectral energy. The energy levels determined for the central wavelengths of the spectral bands $E \lambda_{\text {centr }}(\mathrm{eV})$ were calculated as well.

An occurrence frequency of significant differences was evaluated when ensembles were compared in every experiment with hidden and apparent controls. Here, not only individual homeopathic-like preparations, but total sets of all preparations were compared with both controls, as well as hidden control ensembles were compared with ensembles of apparent control. Furthermore, a significance of difference between frequencies of occurrence was found in regard to the hidden control and apparent controls in every experiment as well as in the total score by means of the1-sided"Difference between 2 proportions" test (STATISTICA Advanced, StatSoft., Basic statistics and tables).

\section{Results}

\subsection{Infrared Absorption Spectra}

The dependences of far infrared transmission coefficients (T\%) on wavelengths (spectral files) for all preparations tested in three experiments are shown in the Supplement Files S1_1_08092016_File.xls, S1_1_15032017_File.xls, S1_1_24052017_File.xls corresponding to the date of each experiment. The spectra are presented in the order of numbering the studied samples at step 4 of the experimental procedure i.e., after unsealing the numbering protocol. The results of the comparative analysis of spectral files by means of the MannWhitney test with Bonferroni's multiple comparison correction in accordance with Equation (1) are shown in the Supplementary Files S1_2_08092016_File.xls, S1_2_15032017_File.xls, 
S1_2_24052017_File.xls corresponding to the date of each experiment. A summary of this comparative analysis is shown in Table 1 for all experiments.

Table 1. Bands of far infrared transmission spectra with significant distinctions between ensembles of homeopathic-like preparations and hidden control "hc"/apparent control " $\mathrm{K}$ ".

\begin{tabular}{|c|c|c|c|c|c|}
\hline Compared Preparations (n) & $\lambda_{\text {centr }} \mathbf{c m}^{-1}$ & $\lambda_{\text {centr, }}, \mathrm{nm}$ & $\mathrm{p}_{\text {cum }}$ & $\begin{array}{l}\text { Direction of } \\
\text { Differences }\end{array}$ & $\mathrm{E} \lambda_{\text {centr }}, \mathrm{eV}$ \\
\hline \multicolumn{6}{|c|}{ Experiment 8 September 2016, distilled water, 1609 spectral readings, $\mathrm{p}_{B}=3.1 \cdot 10^{-5}$} \\
\hline hydroplasma (15): hc (14) & 3612.67 & 2768 & $1.287 \times 10^{-6}$ & + & 0.45 \\
\hline lydocaine (15): hc (14) & 2972.31 & 3364 & $2.046 \times 10^{-72}$ & + & 0.37 \\
\hline Epstein-Barr virus (15): hc (14) & 3628.10 & 2756 & $2.773 \times 10^{-5}$ & + & 0.45 \\
\hline alloplant (15): hc (14) & & & ns & & \\
\hline chorionic gonadotropin (15): hc (14) & & & ns & & \\
\hline $\begin{array}{l}\text { hydroplasma + lidocaine + Epstein-Barr virus + } \\
\text { alloplant + chorionic gonadotropin }(75) \text { : hc (14) }\end{array}$ & & & ns & & \\
\hline \multicolumn{6}{|c|}{ Experiment 15.03 .17 , saline, 1117 spectral readings ${ }^{*}, \mathrm{p}_{\mathrm{B}}=4.476 \cdot 10^{-05}$} \\
\hline hydroplasma (15): hc (14) & & & Ns & & \\
\hline \multirow{2}{*}{\multicolumn{6}{|c|}{$\begin{array}{l}4.998 \times 10^{-6} \\
2.536 \times 10^{-6} \\
9.885 \times 10^{-27} \\
3.845 \times 10^{-89} \\
3.411 \times 10^{-55}\end{array}$}} \\
\hline & & & & & \\
\hline lydocaine (15): K (11) & 3045.60 & 3283 & $4.969 \times 10^{-9}$ & + & 0.38 \\
\hline Epstein-Barr virus (14): hc (14) & \multicolumn{5}{|c|}{ ns } \\
\hline Epstein-Barr virus (14): K (11) & \multicolumn{5}{|c|}{ ns } \\
\hline alloplant (15): hc (14) & 1666.50 & 6000 & $5.181 \times 10^{-8}$ & - & 0.21 \\
\hline alloplant (15): K (11) & \multicolumn{5}{|c|}{ ns } \\
\hline chorionic gonadotropin (14): hc (14) & $\begin{array}{l}1022.27 \\
1481.33 \\
2222.00 \\
2407.16 \\
2939.52 \\
\end{array}$ & $\begin{array}{l}9782 \\
6751 \\
4500 \\
4154 \\
3402 \\
\end{array}$ & $\begin{array}{c}3.178 \times 10^{-6} \\
6.251 \times 10^{-37} \\
6.6583 \times 10^{-28} \\
3.748 \times 10^{-29} \\
8.388 \times 10^{-21}\end{array}$ & $\begin{array}{l}+ \\
+ \\
+ \\
+ \\
+\end{array}$ & $\begin{array}{l}0.13 \\
0.18 \\
0.28 \\
0.30 \\
0.36\end{array}$ \\
\hline chorionic gonadotropin (14): K (11) & $\begin{array}{c}975.98 \\
1377.18 \\
2717.70\end{array}$ & $\begin{array}{c}10,246 \\
7261 \\
3680\end{array}$ & $\begin{array}{l}3.147 \times 10^{-5} \\
7.863 \times 10^{-60} \\
1.069 \times 10^{-72}\end{array}$ & $\begin{array}{l}+ \\
+ \\
+\end{array}$ & $\begin{array}{l}0.12 \\
0.17 \\
0.34\end{array}$ \\
\hline hc (14): K (11) & \multicolumn{5}{|c|}{ ns } \\
\hline $\begin{array}{l}\text { hydroplasma + lidocaine + Epstein-Barr virus + } \\
\text { alloplant + chorionic gonadotropin }(74) \text { : hc }(14)\end{array}$ & 3003.17 & 3330 & $2.560 \times 10^{-7}$ & + & 0.37 \\
\hline $\begin{array}{l}\text { hydroplasma + lidocaine + Epstein-Barr virus + } \\
\text { alloplant + chorionic gonadotropin }(74): \mathrm{K}(11)\end{array}$ & $\begin{array}{l}1400.32 \\
1990.54 \\
2731.20\end{array}$ & $\begin{array}{l}7141 \\
5024 \\
3661\end{array}$ & $\begin{array}{l}3.201 \times 10^{-289} \\
2.324 \times 10^{-177} \\
4.639 \times 10^{-195}\end{array}$ & $\begin{array}{l}+ \\
+ \\
+\end{array}$ & $\begin{array}{l}0.17 \\
0.25 \\
0.34\end{array}$ \\
\hline \multicolumn{6}{|c|}{ Experiment 24.05.2017, saline, 1635 spectral readings, $\mathrm{p}_{\mathrm{B}}=3.058 \cdot 10^{-05}$} \\
\hline hydroplasma (15): hc (15) & 3660.89 & 2731 & $4.509 \times 10^{-9}$ & + & 0.45 \\
\hline lydocaine (15): hc (15) & 972.12 & 10,286 & $1.568 \times 10^{-5}$ & - & 0.12 \\
\hline Epstein-Barr virus (14): hc (15) & & & ns & & \\
\hline alloplant (15): hc (15) & & & ns & & \\
\hline hchorionic gonadotropin (15): hc (15) & & & ns & & \\
\hline $\begin{array}{l}\text { hydroplasma + lidocaine + Epstein-Barr virus + } \\
\text { alloplant + chorionic gonadotropin }(74) \text { : hc (15) }\end{array}$ & $\begin{array}{l}1672.28 \\
3658.96\end{array}$ & $\begin{array}{l}5979 \\
2733\end{array}$ & $\begin{array}{l}2.513 \times 10^{-6} \\
3.142 \times 10^{-7}\end{array}$ & $\begin{array}{l}+ \\
+\end{array}$ & $\begin{array}{l}0.21 \\
0.45\end{array}$ \\
\hline
\end{tabular}

ns-non significant, $(+)$ direction of differences-the 1-st preparation in pair is significantly higher than the 2-nd preparation by the amplitude of spectral readings, (-) direction of differences-the 2-nd preparation in pair is significantly higher than the 1-st preparation by the amplitude of spectral readings, * shortened band of analysis $3100-948 \mathrm{~cm}^{-1}$. 
Note that the experimental procedures varied within the limits of the analysis band, since initially the idea of their combined analysis was not considered.

A part of $\lambda_{\text {centr }}$ values lie on the boundaries of the water absorption band, seen between 3600 and $3000 \mathrm{~cm}^{-1}$. However, the values of transmission coefficient $\mathrm{T} \%$ are in the limits of dynamic range of the apparatus [12].

Significant differences with hidden and apparent controls were revealed in the transmission spectra of some homeopathic-like preparations in far infrared range 2700-10,300 nm (Table 1). Bands with significant differences (sets of adjacent spectral readings) are characterized by significant differences from the spectra of hidden/apparent controls according to the Mann-Whitney U-test. Moreover, the directions of differences in the spectra of the pairs of preparations are predominantly the same. The statistical significance of the differences in the spectra within the identified bands was estimated as sufficient according to the criterion of multiple Bonferroni comparisons Equation (1). Thus, the statistical significance of spectral differences between homeopathic-like preparations and hidden/apparent controls, found in some of studied preparations, may be treated as reliable.

For two preparations of hydroplasma and lydocaine, the differences with apparent/hidden control are observed in all three independent experiments (Table 1). However, for the remaining three preparations, any reproducibility of the results was not found. We cannot yet comment on the physical nature of the poor reproducibility in this case. As for the similarity of the experiments (Table 1) by the spectral regions where significant differences between homeopathic-like preparations and controls are revealed, the energies of central wavelengths $\mathrm{E} \lambda_{\text {centr }}$ for the bands of transmission spectra in most cases $(12 / 14)$ contain components $0.34-0.38 \mathrm{eV}$ and $0.45 \mathrm{eV}$.

\subsection{Terahertz Spectra}

The dependences of terahertz transmission and refractive indices on wavelength (spectral files) for all preparations tested in three experiments are shown in the Supplement Files S2_1_02042018_File.xls, S2_1_24052018_File.xls, S2_1_25122018_File.xls corresponding to the date of each experiment. Results of comparative analysis of spectral files by means of the Mann-Whitney test with Bonferroni's multiple comparison correction in accordance with Equation (1) are shown in the Supplementary Files S2_2_02042018_File.xls, S2_2_24052018_File.pdf, S2_2_25122018_File.pdf corresponding to the date of each experiment. A summary of this comparative analysis is shown in Table 2 for each experiment.

Note that in the experiment conducted on 2 April 2018, only four samples of homeopathiclike preparations of each of the three tested parent substances were prepared. It was reasonable to evaluate only the group differences between the total ensemble of samples lydocaine + euphyllin + analgin $(n=12)$ and the ensemble of samples of the hidden control "hc" ( $\mathrm{n}=12)$.

The transmission spectra at the far end of the range show a sharp decrease in amplitudes. However, the dynamic range between the maximum and minimum values does not exceed 1/0.03-30 dB, which is clearly within the dynamic range of the receiving sensors of the spectrometer.

In some homeopathic-like preparations, significant differences of transmission and refraction spectra in the terahertz (submillimeter) diapason $0.08-0.5 \mathrm{~mm}$ were found in relation to hidden, and mostly apparent, controls. The spectral bands revealed with significant differences (sets of adjacent spectral readings) were characterized by significant differences of homeopathic-like preparations from the controls according to the MannWhitney U-test with Bonferroni corrections for multiple comparisons in accordance with Equation (1). Thus, the statistical significance of spectral differences between homeopathiclike preparations and hidden/apparent control found for some of the studied preparations seems reliable. 
Table 2. Bands of terahertz spectra (transmission and refraction) with significant distinctions between ensembles of homeopathic-like preparations and hidden control "hc" /apparent control "K".

\begin{tabular}{|c|c|c|c|c|c|}
\hline Compared Preparations (n) & $\lambda_{\text {centr }} \mathbf{c m}-1$ & $\lambda_{\text {centr }}, \mathbf{n m}$ & $\mathbf{p}_{\text {cum }}$ & $\begin{array}{l}\text { Direction of } \\
\text { Differences }\end{array}$ & $\mathrm{E} \lambda_{\text {centr }}, \mathrm{eV}$ \\
\hline \multicolumn{6}{|c|}{ Experiment2 April 2018, saline, $\mathrm{p}_{\mathrm{B}}=1.683 \times 10^{-4}$} \\
\hline \multicolumn{6}{|c|}{ Transmission } \\
\hline lydocaine +euphyllin+analgin (12): hc (12) & 118.25 & 84,569 & $1.953 \times 10^{-5}$ & + & 0.015 \\
\hline \multicolumn{6}{|c|}{ Refraction } \\
\hline lydocaine + euphyllin + analgin (12): hc (12) & & & ns & & \\
\hline \multicolumn{6}{|c|}{ Experiment 24 May 2018, saline, $\mathrm{p}_{\mathrm{B}}=1.683 \times 10^{-4}$} \\
\hline \multicolumn{6}{|c|}{ Transmission } \\
\hline lydocaine (12): hc (12) & & & ns & & \\
\hline lydocaine (12): K (12) & 56.31 & 177,595 & $7.262 \times 10^{-20}$ & - & 0.007 \\
\hline euphyllin (12): hc (12) & & & ns & & \\
\hline euphyllin (12): K (12) & & & ns & & \\
\hline analgin (12): hc (12) & & & ns & & \\
\hline analgin (12): K (12) & 106.58 & 93,823 & $8.692 \times 10^{-11}$ & + & 0.013 \\
\hline hc (12): K (12) & & & ns & & \\
\hline lydocaine + euphyllin + analgin (36): K (12) & 56.71 & 176,335 & $8.451 \times 10^{-15}$ & - & 0.007 \\
\hline lydocaine + euphyllin + analgin (36): hc (12) & & & ns & & \\
\hline \multicolumn{6}{|c|}{ Refraction } \\
\hline lydocaine (12): hc (12) & & & ns & & \\
\hline lydocaine (12): K (12) & & & ns & & \\
\hline euphyllin (12): hc (12) & & & ns & & \\
\hline euphyllin (12): K (12) & 107.79 & 92,773 & $1.936 \times 10^{-5}$ & - & 0.013 \\
\hline analgin (12): hc (12) & & & ns & & \\
\hline analgin (12), K (12) & 111.01 & 90,084 & $6.958 \times 10^{-44}$ & - & 0.014 \\
\hline hc (12): K (12) & & & ns & & \\
\hline lydocaine + euphyllin + analgin (36): K (12) & 107.79 & 92,773 & $8.264 \times 10^{-13}$ & - & 0.013 \\
\hline lydocaine + euphyllin + analgin (36): hc (12) & & & $\mathrm{ns}$ & & \\
\hline \multicolumn{6}{|c|}{ Experiment 25 December 2018, saline, $\mathrm{p}_{\mathrm{B}}=1.683 \times 10^{-4}$} \\
\hline \multicolumn{6}{|c|}{ Transmission } \\
\hline lydocaine (15): K (15) & 104.97 & 95,261 & $2.838 \times 10^{-10}$ & - & 0.013 \\
\hline euphyllin (15): K (15) & 111.01 & 90,084 & $3.546 \times 10^{-5}$ & - & 0.014 \\
\hline analgin (15): K (15) & 110.61 & 90,411 & $2.533 \times 10^{-10}$ & - & 0.014 \\
\hline lydocaine + euphyllin + analgin (45): K (15) & $\begin{array}{l}104.17 \\
110.61\end{array}$ & $\begin{array}{l}95,997 \\
90,411\end{array}$ & $\begin{array}{l}6.347 \times 10^{-12} \\
7.828 \times 10^{-11}\end{array}$ & - & $\begin{array}{l}0.013 \\
0.014\end{array}$ \\
\hline \multicolumn{6}{|c|}{ Refraction } \\
\hline lydocaine (15): K (15) & 119.05 & 83,997 & $5.268 \times 10^{-13}$ & + & 0.015 \\
\hline euphyllin (15): K (15) & & & ns & & \\
\hline analgin (15): K (15) & $\begin{array}{c}22.52 \\
119.45\end{array}$ & $\begin{array}{c}443,988 \\
83,714\end{array}$ & $\begin{array}{c}2.302 \times 10^{-93} \\
1.039 \times 10^{-6}\end{array}$ & $\begin{array}{l}+ \\
+\end{array}$ & $\begin{array}{l}0.003 \\
0.015\end{array}$ \\
\hline lydocaine + euphyllin + analgin (45): K (15) & $\begin{array}{c}16.89 \\
119.45\end{array}$ & $\begin{array}{c}591,985 \\
83,714\end{array}$ & $\begin{array}{l}1.075 \times 10^{-4} \\
3.447 \times 10^{-6}\end{array}$ & $\begin{array}{l}+ \\
+\end{array}$ & $\begin{array}{l}0.002 \\
0.015\end{array}$ \\
\hline
\end{tabular}

ns-non significant, (+) direction of differences-the 1-st preparation in pair is significantly higher than the 2-nd preparation by the amplitude of spectral readings, (-) direction of differences- the 2-nd preparation in pair is significantly higher than the 1-st preparation by the amplitude of spectral readings. 


\subsection{Dynamic Light Scattering Spectral Parameters}

The spectral parameters of dynamic light scattering for all preparations tested in the three experiments as well as the results of the comparative analysis of spectral parameters by means of the Mann-Whitney test are shown in the Supplement Files S3_03022018_File.pdf, S3_22092017_File.pdf, S3_22112017_File.pdf corresponding to the date of each experiment. The results of comparative analysis are shown in Table 3 for each experiment.

Table 3. Significance $\mathrm{p}_{\text {cum }}$ (direction) of the differences in ensembles of homeopathic-like preparations and hidden control "hc" / apparent "K" control by spectral parameters of dynamic light scattering.

\begin{tabular}{|c|c|c|c|c|}
\hline Compared Preparations (n) & $\%$ Intensity & Size & $\begin{array}{c}\text { Standard } \\
\text { Deviation (Size) }\end{array}$ & Band, nm \\
\hline \multicolumn{5}{|c|}{ Experiment 3 February 2018, saline, $\mathrm{p}_{\mathrm{B}}=0.0125$} \\
\hline lydocaine (9): hc (10) & ns & ns & ns & $4-1765$ \\
\hline lydocaine (9): K (12) & $0.002(-)$ & ns & ns & $4-1765$ \\
\hline euphyllin (12): hc (10) & ns & ns & ns & $4-1765$ \\
\hline euphyllin (12): K (12) & $0.00001(-)$ & ns & ns & $4-1765$ \\
\hline analgin (12): hc (10) & ns & ns & ns & $4-1765$ \\
\hline analgin (12): K (12) & $0.0009(-)$ & ns & ns & $4-1765$ \\
\hline hc (10): K (12) & $0.004(-)$ & ns & ns & $4-1765$ \\
\hline lydocaine +euphyllin +analgin (33): hc (10) & ns & ns & ns & $4-1765$ \\
\hline lydocaine + euphyllin + analgin (33): K (12) & $0.000004(-)$ & ns & ns & $4-1765$ \\
\hline \multicolumn{5}{|c|}{ Experiment 22 November 2017, saline, $\mathrm{p}_{\mathrm{B}}=0.0125$} \\
\hline lydocaine (11): hc (7) & ns & ns & ns & $7-1369$ \\
\hline lydocaine (11): K (19) & ns & ns & ns & $7-1369$ \\
\hline euphyllin (7): hc (7) & ns & ns & ns & $7-1369$ \\
\hline euphyllin (7): K (19) & ns & ns & ns & $7-1369$ \\
\hline analgin (9): hc (7) & ns & ns & ns & $7-1369$ \\
\hline analgin (9): K (19) & ns & $0.0001(-)$ & $0.0009(-)$ & $7-1369$ \\
\hline hc (7): K (19) & ns & $0.0004(-)$ & $0.002(-)$ & $7-1369$ \\
\hline lydocaine + euphyllin + analgin (27): hc (7) & ns & ns & ns & $7-1369$ \\
\hline lydocaine + euphyllin + analgin (27): K (19) & ns & $0.0003(-)$ & $0.0007(-)$ & $7-1369$ \\
\hline \multicolumn{5}{|c|}{ Experiment 22 September 2017, distilled water, $\mathrm{p}_{\mathrm{B}}=0.0125$} \\
\hline lydocaine (11): hc (10) & $0.006(-)$ & ns & ns & $40-1360$ \\
\hline uranium (9): hc (10) & ns & ns & ns & $40-1360$ \\
\hline cerebral spinal fluid (9): hc (10) & ns & ns & ns & $40-1360$ \\
\hline lydocaine + uranium +cerebral spinal fluid (29): hc (10) & $\mathrm{ns}$ & $\mathrm{ns}$ & ns & $40-1360$ \\
\hline
\end{tabular}

ns-non significant, (-) direction of differences-the 2-nd preparation in pair is significantly higher than the 1-st preparation by the value of current spectral parameter.

Note that the procedures of experiments slightly varied in the composition of the preparations and analyzed bands, because initially, the idea of combining them was not considered.

The following spectral parameters were then analyzed: “\% Intensity" - percentage of the intensity of the first scattering peak; "Size" - size of the scatterer according to the first peak; and "Standard Deviation (Size)"—standard deviation of the "Size" parameter. 
The dynamic range for the dimensions of scatterers (Size) under the analysis did not exceed $55 \mathrm{~dB}$, and the considered scattering intensity peaks (\% Intensity) amounted to $26 \mathrm{~dB}$, which clearly fits to the dynamic range of the receiving paths of the spectrometer.

A feature of the experiments with dynamic light scattering was the rejection of a relatively large number of spectra of samples in which there were either no scattering peaks at all, or their size were beyond the boundaries of the accepted ranges (see Supplementary Files S3_File).

When evaluating paired statistical differences between spectral parameters of ensembles by the Mann-Whitney U-test with Bonferroni correction for multiple comparisons, the condition $\mathrm{p}<\mathrm{p}_{\mathrm{B}}=0.05 / 4=0.0125$ was used.

In the spectra of dynamic light scattering (ranges 4-1765 nm, 7-1369 nm, 40-1360 nm, in various experiments), some of the homeopathic-like preparations showed significant differences with hidden, and mostly, with the apparent control. The spectral parameters of the preparations are characterized by significant differences from the controls by the MannWhitney U-test with Bonferroni corrections for multiple comparisons. Thus, the statistical significance of differences in the spectral parameters of homeopathic-like preparations and hidden/apparent control found in some of the studied preparations seems reliable.

The differences in the spectral parameters with the controls are unidirectional, but they diverge in the groups of spectral parameters-for a part of preparations in "\% Intensity", while for others in "Size" and "St Dev (Size)". However, the scattering intensity (\% Intensity) is determined not only by the concentration of scatterers, but also by their size (Size); therefore, a correlation between these two spectral parameters can be expected.

\subsection{Frequency of Occurrence of Spectral Differences in Preparations under Study in Relation toHidden and Apparent Controls}

Among the results of all experiments, performed in three spectral diapasons, the frequency of occurrence of significant spectral differences between homeopathic-like preparations and hidden "hc" /apparent " $\mathrm{K}$ " controls were found.

Based on this observation, let us consider homeopathic-like preparations as common objects, not specific to the parent substances. It is noteworthy for increasing the statistical basis of analysis. It is reasonable to gather all blinded preparations (individual homeopathic-like preparations, the total ensembles of homeopathic-like preparations, hidden controls), prepared in the same way, to compare with the apparent (not blinded) control " $\mathrm{K}$ ". Alternatively, blinded preparations (individual homeopathic-like preparations and the total ensembles of homeopathic-like preparations) should be compared with the hidden (blinded) control "hc". That is why such comparisons were made, and the data are presented in Tables 1-3.

The results of the supposed evaluation are shown in Table 4, where the first column represents the frequencies of occurrence of spectral differences when comparing blinded homeopathic-like preparations with the blinded (hidden) control, and the second column represents the frequencies of occurrence of spectral differences when comparing all blinded preparations with the apparent (not blinded) control.

It follows from Table 4 that the frequency of occurrence of statistically significant differences between the blinded homeopathic-like preparations and the apparent control " $\mathrm{K}$ " is significantly higher than the frequency of occurrence of statistically significant differences between the blinded homeopathic-like preparations and the hidden control "hc" in terahertz spectra ( 0.72 vs. $0.1, p=0.0021)$ and in spectral parameters of dynamic light scattering ( 0.8 vs. $0.083, p=0.0014)$. Only in far infrared transmission spectra did these differences turn out to be statistically insignificant $(0.57 \mathrm{vs} .0 .5, \mathrm{p}>0.05)$. The total score of the frequencies of occurrence in all three diapasons significantly differs once again in favor of apparent control "K" (0.71 vs. $0.275, \mathrm{p}=0.0002)$. 
Table 4. Frequencies of occurrence of significant spectral distinctions of homeopathic-like preparations with the hidden control "hc" and the apparent control " $\mathrm{K}$ " and significance of differences in the frequencies.

\begin{tabular}{|c|c|c|}
\hline Date of Experiment & $\begin{array}{l}\text { Blinded Homeopathic-like } \\
\text { Preparations with Hidden Control "hc" }\end{array}$ & $\begin{array}{l}\text { All Blinded Homeopathic-like } \\
\text { Preparations with Apparent Control } \\
\text { "K" }\end{array}$ \\
\hline \multicolumn{3}{|c|}{ Far infrared spectral transmission } \\
\hline 8 September 2016 & 3 from 6 & - \\
\hline 24 May 2017 & 3 from 6 & - \\
\hline 15 March 2017 & 3 from 6 & 4 from 7 \\
\hline Incidences & $9 / 18=0.5$ & $4 / 7=0.57$ \\
\hline Significance of difference & \multicolumn{2}{|c|}{ ns } \\
\hline \multicolumn{3}{|c|}{ Terahertz spectral transmission and refraction } \\
\hline 2 April 2018 & $\begin{array}{l}1 \text { from } 1 \text { (transmission), } \\
0 \text { from } 1 \text { (refraction) }\end{array}$ & - \\
\hline 24 May 2018 & $\begin{array}{l}0 \text { from } 4 \text { (transmission), } \\
0 \text { from } 4 \text { (refraction) }\end{array}$ & $\begin{array}{l}3 \text { from } 5 \text { (transmission), } \\
3 \text { from } 5 \text { (refraction) }\end{array}$ \\
\hline 25 December 2018 & - & $\begin{array}{l}4 \text { from } 4 \text { (transmission), } \\
3 \text { from } 4 \text { (refraction) }\end{array}$ \\
\hline Incidences & $1 / 10=0.1$ & $13 / 18=0.72$ \\
\hline Significance of difference & \multicolumn{2}{|c|}{$\mathrm{p}=0.0021$} \\
\hline \multicolumn{3}{|c|}{ Spectral parameters of dynamic light-scattering } \\
\hline 3 February 2018 & 0 from 4 & 5 from 5 \\
\hline 22 September 2017 & 1 from 4 & - \\
\hline 22 November 2017 & 0 from 4 & 3 from 5 \\
\hline Incidences & $1 / 12=0.083$ & $8 / 10=0.8$ \\
\hline Significance of difference & \multicolumn{2}{|c|}{$\mathrm{p}=0.0014$} \\
\hline Total incidences (in all 3 diapasons) & $11 / 40=0.275$ & $25 / 35=0.71$ \\
\hline Significance of difference & \multicolumn{2}{|c|}{$p=0.0002$} \\
\hline
\end{tabular}

\section{Discussion}

So, we performed nine independent blinded experiments with homeopathic-like preparations of several parent substances, which involved measuring far infrared transmission spectra $(n=3)$, terahertz transmission and refraction spectra $(n=3)$, and dynamic light scattering spectral parameters $(n=3)$. Statistically significant spectral differences of homeopathic-like preparations with the apparent control $(25 / 35=0.71)$ and the hidden control $(11 / 40=0.275)$ were revealed (Tables $1-4)$.

The first question that should be discussed concerns whether the identification of these significant differences is a consequence of instrumental bias and/or errors.

All investigated preparations (homeopathic-like preparations as well as hidden/apparent control samples) were measured in every experiment in the same metering set of the same apparatus, but in random order. Therefore, it seems possible to neglect the bias in the results due to the drift of the apparatus characteristics.

At the same time, variations in the assembling (washing, drying) of the measuring setup, as well as in homeopathic-like preparations-producing procedure, can lead to random error. However, this kind of error is taken into account by the Mann-Whitney U-test with Bonferroni corrections for multiple comparisons, used to establish the statistical significance of the differences in spectra between ensembles of preparations. 
Thus, identified distinctions in spectra of homeopathic-like preparations and hidden/apparent controls cannot be attributed to any spectral apparatus bias or random error.

As for the dynamic range of the evaluated spectral signals, in all three types of spectral measurements (diapasons), it did not exceed the available dynamic range of receiving tracts of spectrometers. Thus, spectral measurements were carried out at sufficient levels of signal/noise ratio, which excluded the influence of background noise of equipment on the obtained results [12].On the other hand, it is not surprising that the identified portions of spectra, characterized by the most prominent difference in homeopathic-like preparations and hidden/apparent control, lie in the areas of maximal water absorption (in neighborhood of minima of transmission and refractive indexes), because in these regions, intensive interaction of emission of spectrometers with a molecular structure of water may be expected [12].

\subsection{Physical Interpretation of The Results in Every Diapason}

When assessing the results physically, one need to keep in mind that we are really considering the result of two actions on the samples-one of them is associated with producing homeopathic-like preparations, while the second one is bonded with illumination of the sample by the spectrometer radiation when measuring the spectrum. This is the main problem of the physical interpretation of our findings.

Another problem is a time delay between producing homeopathic-like preparations and measuring their spectra. This time lag could probably weaken physical changes involved in the producing procedure. However, similar time lag (units-tens of days) is typical for using homeopathic-like preparations in the curative practice of CAM. Thus, our time-expanded study of properties of these preparations seems quite pertinent.

\subsubsection{Far Infrared Spectra}

The indicated energy value turns out to be close to the maxima of the transmission spectra $0.34-0.38$ and $0.45 \mathrm{eV}$ that we have identified (Table 1), in which statistically significant differences between homeopathic-like preparations and hidden/apparent controls were found. Surprisingly, these differences for all studied preparations and in all three experiments are predominantly unidirectional (sign + ) and indicate greater transmission, i.e., less absorption, of infrared radiation of the spectrometer by homeopathic-like preparations than by controls.

\subsubsection{Terahertz Spectra}

It should be considered in the physical interpretation of the results that the energies of the central wavelengths of the detected spectral bands with significant differences of the homeopathic-like preparations from the controls (Table 2) are very small and lie outside the obvious vibrational resonances of water molecules or their supramolecular formations. However, in this range, the water dipole molecules attempt to continuously reorient in an oscillating electromagnetic field. The ease of the movement depends on the viscosity and the mobility of the electron clouds. In water, these rely on the strength and extent of the hydrogen-bonded network [14]. Thus, the supramolecular organization of water solutions can make a certain contribution to the obtained spectra.

According to the results of all three experiments in this diapason, the directions of the revealed differences in the transmission spectra are predominantly (7/9) unidirectional (sign-). It seems likely that the values of spectral readings in the transmission spectra of the apparent/hidden controls are higher than those of homeopathic-like preparations. Therefore, homeopathic-like preparations absorb more radiation of the spectrometer than the control samples. This may be a consequence of "the strength and extent of the hydrogen-bonded network" [14], i.e., more developed supramolecular formations, or higher organization of the microstructure of solutions in homeopathic-like preparations. However, in accordance with the main problem of physical interpretation, formulated above, the question of "producing" or "measuring" is behind this effect. 


\subsubsection{Dynamic Light Scattering Spectra}

In physical interpretation of the results, it may be assumed that the main laser radiation scatterers are gas nanobubbles stabilized by ions (bubstons) $[15,16]$, which are estimated here from 200 to $450 \mathrm{~nm}$ in size by medians. It is clear that unidirectionality (sign-) is seen for all significant differences with control assemblies in spectral parameters. Therefore, in homeopathic-like preparations, the associated spectral parameters (intensity and/or size of scatterers) should be lower than in controls. Based on considering the two types of influence on the samples, one of which is associated with producing homeopathic-like preparations, and the second one due to the illumination of the sample with laser radiation when measuring the spectrum, the main problem is the following: What of these effects may be responsible for the revealed differences in the spectral parameters of samples? This question still remains open for this diapason.

\subsection{Physical Interpretation of the Difference in the Frequency of Distinctions Occurrence in Relation to Hidden and Apparent Controls}

As follows from Table 4, the frequency of occurrence of statistically significant spectral distinctions between homeopathic-like preparations and the apparent control " $\mathrm{K}$ ", as a rule, is significantly higher than the frequency of occurrence of statistically significant spectral distinctions between homeopathic-like preparations and the hidden control "hc". The only exception is seen for the far infrared range, despite the most time lags between producing the samples and measuring their spectra. Judging the total score of the frequencies of occurrence, the spectral distinctions of blinded preparations with the apparent (not blinded) control $(25 / 35)$ are seen more frequently than those with the hidden (blinded)control (11/40).

We can probably assume that more sophisticated physical mechanisms, inaccessible to the modern instrumental base in terms of sensitivity and accuracy, could be considered as a result of homeopathic-like preparations (especially in far infrared diapason, where the evident "observer effect" was not found). However, judging from the results obtained here, these mechanisms should be largely masked by the effect of the observer expectations. Obviously, the key factor leading to the realization of the "observer effect" should be the illumination of the studied preparations by spectrometer radiation. However, such illumination is possible in nature without any spectrometer. It was evaluated recently [17] that a human body is bombarded by 1 sextillion per each second of only sun-emitted photons lying from ultraviolet to infrared diapason. Thus, we "bathe" in external electromagnetic illumination.

\subsection{Limitations and Future Works}

The obtained results and conclusions on homeopathic-like preparations suffer from a limited volume of experiments, small statistical basis, and an absence of strict synchronization in measurements with hidden/apparent controls. It would be necessary to extend the study with larger volumes of preparations, using synchronous combinations of hidden and apparent controls in every experiment. It is also desirable to minimize the time lag between producing the homeopathic-like preparations and measuring their spectra.

\section{Conclusions}

1. In the analysis of nine independent blind studies of aqueous homeopathic-like preparations of several parent substances carried out by measuring infrared transmission spectra, terahertz transmission and refraction spectra, and dynamic light scattering, statistically significant spectral differences are found in some preparations with the apparent control (the frequency of occurrence 25/35) and the hidden control (the frequency of occurrence 11/40).

2. The specificity of the differences between homeopathic-like preparations and apparent/hidden controls neither in the parent substances nor in the characteristic wavelengths or spectral parameters is observed. 
3. The revealed dominance in the frequency of differences occurrence in any homeopathiclike preparation (without separation by parent substances) with regard to the apparent control being above the frequency of differences occurrence for the hidden control can be treated as involving most of the changes to the samples not by the homeopathic-like preparations-producing procedure, but by the spectral measuring process under illumination with spectrometer radiation.

4. If more sophisticated physical mechanisms, inaccessible to the modern instrumental base in terms of sensitivity and accuracy, will be assumed responsible for homeopathic-like preparation producing, they would be largely masked by the "observer effect" resulting in a bias for a non-blind study as well as in random (non-directional) error for the blind one.

Supplementary Materials: The following are available online at https:/ /www.mdpi.com/article / 10.3390/w13111475/s1, The list of these files is: S1_1_08092016_File.xls; S1_1_24052017_File.xls; S1_1_24052017_File.xls, S1_2_08092016_File.xls, S1_2_15032017_File.xls, S1_2_24052017_File.xls, S2_1_02042018_File.xls; S2_1_24052018_File.xls; S2_1_25122018_File.xls; S2_2_02042018_File.xls; S2_2_24052018_File.pdf; S2_2_25122018_File.pdf; S3_03022018_File.pdf; S3_22092017_File.pdf; S3_22112017_File.pdf.

Author Contributions: Conceptualization, V.K. and T.C.; methodology, V.K., N.B., and T.C.; validation, V.K., N.B., and S.Z., formal analysis, V.G.; investigation, N.B. and A.U.; data curation, V.G. and R.G.; writing - original draft preparation, V.K.; writing-review and editing, T.C. and N.B. All authors have read and agreed to the published version of the manuscript.

Funding: The research was partially funded by the State research program of Russian academy of sciences (state registration project No. AAAA-A20-120031890011-8).

Institutional Review Board Statement: Not applicable.

Data Availability Statement: Data supporting reported results can be found in Supplementary Files attached.

Acknowledgments: Authors acknowledge N. Penkov and V. Kostin for assistance in performing spectral measurements.

Conflicts of Interest: The authors declare no conflict of interest.

\section{References}

1. Shang, A.; Huwiler-Muntener, K.; Nartey, L.; Juni, P.; Dorig, S.; Sterne, J.A.C.; Pewsner, D.; Egger, M. Are the clinical effects of homoeopathy placebo effects? Comparative study of placebo-controlled trials of homoeopathy and allopathy. Lancet 2005, 366, 726-732. [PubMed]

2. Righetti, M.; Von Ammon, K.; Malchow, H.; Frei-Erb, M.; Bläuer, F.; Etter, G. Comments on the Article ‘Beliefs, Endorsement and Application of Homeopathy Disclosed: A Survey among Ambulatory Care Physicians' by Markun et al. Complement. Med. Res. 2018, 25, 417-418. [CrossRef] [PubMed]

3. Klein, S.D.; Würtenberger, S.; Wolf, U.; Baumgartner, S.; Tournier, A. Physicochemical Investigations of Homeopathic Preparations: A Systematic Review and Bibliometric Analysis_Part 1. J. Altern. Complement. Med. 2018, 24, 409-421. [CrossRef] [PubMed]

4. Bunkin, N.F.; Shkirin, A.V.; Penkov, N.V.; Chirikov, S.N.; Ignatiev, P.S.; Kozlov, V.A. The physical nature of mesoscopic inhomogeneities in highly diluted aqueous suspensions of protein particles. Phys. Wave Phenom. 2019, 27, 102-112. [CrossRef]

5. Konovalov, A.I.; Ryzhkina, I.S. Formation of nanoassociates as a key to understanding of physicochemical and biological properties of highly dilute aqueous solutions. Russ. Chem. Bull. 2014, 63, 1-14. [CrossRef]

6. Chaplin, M.F. The Memory of Water: An overview. Homeopathy 2007, 96, 143-150. [CrossRef] [PubMed]

7. Towsey, M.W.; Hasan, M.Y. Homoeopathy—a biophysical point of view. Br. Homeopath. J. 1995, 84, 218-228. [CrossRef]

8. Thomas, Y.; Schiff, M.; Belkadi, L.; Jurgens, P.; Kahhak, L.; Benveniste, J. Activation of human neutrophils by electronically transmitted phorbolmyristate acetate. Med. Hypotheses. 2000, 54, 33-39. [CrossRef] [PubMed]

9. Montagnier, L.; Aïssa, J.; Ferris, S.; Montagnier, J.L.; Lavallée, C. Electromagnetic signals are produced by aqueous nanostructures derived from bacterial DNA sequences. Interdiscip Sci. 2009, 1, 81-90. [CrossRef] [PubMed]

10. Klein, S.D.; Tournier, A.; Würtenberger, S.; Wolf, U.; Baumgartner, S. Physicochemical investigations of homeopathic potencies: A systematic review of the literature. Eur. J. Integr. Med. 2016, 8, 1-66. [CrossRef]

11. Jonas, W.B.; Ives, J.A.; Rollwagen, F.; Denman, D.W.; Hintz, K.; Hammer, M.; Crawford, C.; Henry, K. Can specific biological signals be digitized? FASEB J. 2006, 20, 23-28. [CrossRef] [PubMed] 
12. Korenbaum, V.; Chernysheva, T.; Galay, V.; Galay, R.; Ustinov, A.; Vladislav, K.; Zakharkov, S. On the reliability of spectral evidences of electronic copying phenomenon used to produce homeopathic-like preparations in complementary medicine. Water 2019, 11, 1-13. [CrossRef]

13. Penkov, N.; Yashin, V.; Fesenko, E., Jr.; Manokhin, A.; Fesenko, E. A study of the effect of a protein on the structure of water in solution using terahertz time-domain spectroscopy. Appl. Spectrosc. 2018, 72, 257-267. [CrossRef] [PubMed]

14. Available online: www.idc-online.com/technical_references/pdfs/chemical_engineering/Water_absorption_spectrum.pdf (accessed on 23 May 2021).

15. Bunkin, N.F.; Bunkin, F.V. Bubston structure of water and electrolyte aqueous solutions. Phys. Usp. 2016, 59, 846-865. [CrossRef]

16. Yurchenko, S.O.; Shkirin, A.V.; Ninham, B.W.; Sychev, A.A.; Babenko, V.A.; Penkov, N.V.; Kryuchkov, N.P.; Bunkin, N.F. Ionspecific and thermal effects in the stabilization of the gas nanobubble phase in bulk aqueous electrolyte solutions. Langmuir 2016, 32, 11245-11255. [CrossRef] [PubMed]

17. Driver, S.P.; Andrews, S.K.; Davies, L.; Robotham, A.S.G.; Wright, A.H.; Windhorst, R.A.; Cohen, S.; Emig, K.; Jansen, R.A.; Dunne, L. Measurements of extragalactic background light from the far uv to the far ir from deep ground-and space-based galaxy counts. Astrophys. J. 2016, 827, 108. [CrossRef] 